\title{
Eficiencia De La Terapia Manual Omt Kaltenborn- Evjenth En La Marcha Del Paciente Geriátrico Diabético
}

\author{
Casales Orozco Georgina, \\ Martínez Guillén Valeria Irais, \\ Estudiante de la Licenciatura en Fisioterapia,
} Facultad de Enfermería, Universidad Autónoma de Querétaro, México

Xeque Morales Ángel Salvador, Maestro en Ciencias, Facultad de Enfermería, Universidad Autónoma de Querétaro, México

García Medina Nadia Edith, Dra. en Ciencias Neurológicas, Facultad de Enfermería, Universidad Autónoma de Querétaro, México

\section{Resumen}

Introducción: La diabetes afecta a la población geriátrica ocasionando alteraciones funcionales, desequilibrio, atrofias y deformidades del pie, generando patrones compensatorios. La terapia manual OMT KaltenbornEvjenth evalúa y trata alteraciones articulares, y es eficaz para la hipomovilidad y la disminución del dolor por medio de tracciones, compresiones y movilizaciones articulares. Objetivo: Determinar la eficacia de la terapia manual OMT Kaltenborn-Evjenth en la marcha del paciente geriátrico diabético. Método: A 28 pacientes diabéticos se les aplicó la prueba de Batería Breve de Rendimiento Físico (por sus siglas en inglés, SPPB) para determinar limitación funcional de la marcha (LFM) y el tipo de la misma, se les aplicó 8 sesiones de terapia manual OMT Kaltenborn-Evjenth para tobillo y pie, realizando deslizamientos plantares, dorsales del escafoides, cuboides, tracciones distales de calcáneo y movilización tibiotarsiana. Tras la última sesión con la prueba SPPB se determinó el cambio del tipo de limitación de la marcha. Resultados: La edad promedio de la población fue de $68 \pm 3.3$ años, predominando el género femenino hasta en un $82 \%$. Al término de las sesiones de terapia manual por el método OMT Kaltenborn-Evjenth, la LFM predominante fue la leve en un $71 \%$ de los pacientes, $18 \%$ moderada, y $11 \%$ mínima. Mostrando notablemente cambios favorables significativos en el tipo de LFM. Conclusión: la terapia manual OMT Kaltenborn-Evjenth es efectiva 
para disminuir la LFM pacientes geriátricos diabéticos.

Parablas clave: Terapia manual, Geriátrico, Diabético, Marcha

\title{
Efficiency of Omt Kaltenborn-Evjenth in the Gait of Diabetic Geriatric Patients
}

\author{
Casales Orozco Georgina, \\ Martínez Guillén Valeria Irais,
}

Estudiante de la Licenciatura en Fisioterapia,

Facultad de Enfermería, Universidad Autónoma de Querétaro, México

Xeque Morales Ángel Salvador,

Maestro en Ciencias, Facultad de Enfermería,

Universidad Autónoma de Querétaro, México

García Medina Nadia Edith,

Dra. en Ciencias Neurológicas, Facultad de Enfermería,

Universidad Autónoma de Querétaro, México

\begin{abstract}
Introduction: Diabetes affects the geriatric population causing mechanical and functional alterations, imbalance, atrophy and deformities of the foot. The limitation of the movement increases the stiffness, the pressure of the plantar support and the difficulty to the adaptation of the march, generating compensatory patterns. Manual therapy through the OMT Kaltenborn-Evjenth technique evaluates and treats joint alterations, and is effective for hypomobility and pain reduction through traction, compression and joint mobilization. Objective: To determine the effectiveness of manual therapy OMT Kaltenborn-Evjenth in the geriatric patient's march with diabetes Method: 28 diabetic patients underwent the Short Physical Performance Battery (SPPB) test to determine the gait limitation (FLG) and its type, 8 sessions of manual therapy OMT Kaltenborn-Evjenth were applied for ankle and foot, performing plantar landslides, dorsal scaphoid, cuboid, distal calcaneal traction and tibiotarsian mobilization. After the last session with the SPPB test, the change in the type of gait limitation was determined. Results: The average age of the population was $68 \pm 3.3$ years, with the female gender prevailing up to $82 \%$. At the end of the manual therapy sessions by the Kaltenborn-Evjenth OMT method, the predominant FLG was mild in $71 \%$ of the patients, $18 \%$ moderate, and $11 \%$ minimal. Significantly showing
\end{abstract}


favorable changes in the type of FLG. Conclusion: Kaltenborn-Evjenth OMT manual therapy is effective in reducing FLG diabetic geriatric patients.

Keywords: Manual therapy, Geriatric, Diabetic, Gait

\section{Introducción}

La marcha bipodal es el medio mecánico de locomoción del ser humano, es decir, translación en la cual el hombre erguido es soportado de manera alternativa por ambas extremidades inferiores. (Sgaravatti et al., 2018) En el envejecimiento ocurren una serie de modificaciones en el sistema músculo-esquelético y en los mecanismos nerviosos centrales y periféricos provocando cambios en el patrón normal de la marcha, que constituyen la marcha senil. La velocidad de marcha menor a $1 \mathrm{~m} / \mathrm{s}$ es un posible indicador de eventos adversos en el adulto mayor aparentemente sano y si la velocidad de marcha disminuye hasta menos de $0,84 \mathrm{~m} / \mathrm{s}$, se puede perder la capacidad de marcha funcional. (Herrero- Larrea, 2017) Por ello, los protocolos de análisis de marcha y postura resultan esenciales en las personas de la tercera edad. En pacientes con diabetes se producen alteraciones biomecánicas y funcionales que provocan desequilibrio muscular, con atrofias y deformidades estructurales secundarias del pie. La limitación de la movilidad articular provoca un aumento de la presión del apoyo plantar y mayor rigidez, con dificultad para la adaptación a la marcha fisiológica y el desarrollo de patrones compensadores de marcha. La limitación de la movilidad articular del pie es de aparición precoz en diabetes mellitus. (Kang et al., 2019)

Los adultos mayores con diabetes tienen una menor velocidad de la marcha, pasos más cortos y una base de sustentación más amplia. Además son más susceptibles al desarrollo de otras enfermedades y por ende polifarmacia, condicionando un incremento en el riesgo de caídas (Secretaría de Salud 2013). Además Prishila et al., (2016) mencionan que la valoración y tratamiento oportuno de las enfermedades de las extremidades inferiores son cruciales para evitar el desarrollo de complicaciones como el pie diabético o amputaciones que le requieren a los sistemas de salud pública un alto costo.

La Batería Breve de Rendimiento Físico es una medida objetiva que permite clasificar el nivel de funcionamiento físico de las personas mayores, la cual consta de tres pruebas: equilibrio, velocidad de la marcha y, levantarse y sentarse en una silla cinco veces (Rodríguez-Mañas et al., 2014). En el test de equilibrio el participante intenta mantener 3 posiciones parado: pies juntos, semitándem y tándem durante 10 segundos cada uno. En el test de velocidad de la marcha, el participante anda a su ritmo habitual una distancia de 4 metros. Este se realiza dos veces y se registra el tiempo más breve. Finalmente, en el test de levantarse y sentarse en una silla cinco veces, el participante se levanta y se sienta en una silla cinco veces, de la forma más rápida posible, y se registra 
el tiempo total empleado. Cada test se puntúa de 0 (peor rendimiento) a 4 (mejor rendimiento) en función del tiempo empleado. Además, se obtiene una puntuación total para toda la batería que es la suma de la de las tres pruebas y que oscila entre 0 y 12. (Lauretani et al., 2019)

En el sistema OMT (Terapia Manipulativa Ortopédica) es una técnica de movilización articular en la que se usan los movimientos translatorios de deslizamiento y tracción en diferentes grados de movimiento para estirar la cápsula articular o los ligamentos retraídos y de esta manera restaurar un adecuado juego articular. El objetivo de la movilización articular es restaurar la función articular normal e indolora (Hammad et al., 2019). Por tal razón, el objetivo del presente estudio fue determinar la eficacia de la terapia OMT Kaltenborn-Evjenth en alteraciones mecánicas del tobillo en el paciente geriátrico diabético con fines de mejorar la marcha.

\section{Material y métodos}

Estudio cuasiexperimental, longitudinal y prospectivo, de mediciones antes y después en pacientes geriátricos diabéticos que acudieron al servicio de Medicina Física y Rehabilitación de dos UMF en la ciudad de Santiago de Querétaro, del 1 de agosto de 2017 a Junio de 2018. Dichos pacientes debían contar con un diagnóstico médico de diabetes mellitus, ser independientes para la marcha, no tener antecedentes de EVC, radiculopatías o prótesis de miembro inferior. Para el tamaño de muestra se aplicó la fórmula para población finita (García-García et al., 2013), la cual arrojó un resultado de 27 pacientes, y la técnica muestral utilizada fue no probabilística por conveniencia. Para las variables cuantitativas los resultados se indicaron con medias y desviación estándar, para las variables cualitativas con frecuencias (porcentajes). La normalidad de la variable Limitación funcional de la marcha fue evaluada con la prueba Shapiro-Wilk y su comparación de los puntos observados al inicio y al final con la prueba T-student con un intervalo de confianza de $95 \%$ y significancia estadística con un valor p<0.050. Se usó el programa informático SPSS para el análisis estadístico. Se otorgó a cada participante una sesión informativa acerca del protocolo de investigación al cual accedieron a formar parte, se midieron y documentaron las variables que serán descritas a continuación. A los pacientes se les realizó la prueba Short Physical Performance Battery (SPPB) para valoración de la marcha la cual es validada en pacientes geriátricos.

\section{Programa de terapia manual}

Se otorgaron ocho sesiones individuales a cada paciente, con una duración de 30 minutos y una frecuencia de dos veces por semana. En la primera sesión los pacientes firmaron el consentimiento informado y se les aplicó el instrumento de recolección de datos y la primera medición de la 
Batería Breve de Rendimiento Físico. En las sesiones restantes se realizó la técnica de terapia manual articular Kaltenborn-Evjenth para articulación de tobillo. Para finalizar, en la última sesión se realizó la medición final para la SPPB. La técnica de terapia manual articular Kaltenborn-Evjenth destinada a articulación de tobillo consiste en realizar maniobras de deslizamiento plantar y dorsal del escafoides, deslizamiento de cuboides, tracción distal del calcáneo y movilización de la articulación tibiotarsiana (Kaltenborn et al., 2001).

\section{Resultados}

La población estudiada incluyó 28 pacientes, de las cuales el género femenino fue el predominante con un total de 23 mujeres $(82 \%)$ y 5 hombres (18\%), con una edad promedio de 68 años. Todos con diagnóstico previo de diabetes mellitus controlados por su médico. En el puntaje inicial se obtuvo una media $5.6 \pm 1.3$, se observó un aumento en la puntuación final de $7.9 \pm$ 1.4, como se puede observar en la Figura 1.

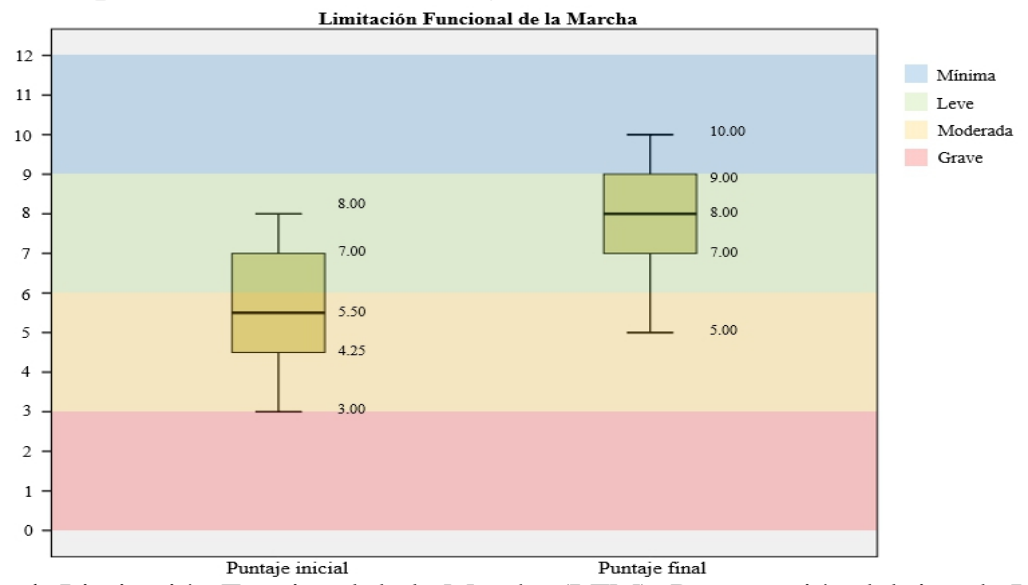

Figura 1. Limitación Funcional de la Marcha (LFM). Presentación del tipo de LFM en pacientes geriátricos diabéticos al inicio (puntaje inicial) y al final (puntaje final) de haber tomado ocho sesiones de terapia manual OMT Kaltenborn-Evjenth. Las barras horizontales representan el tipo de LFM como mínima (azul), leve (verde), moderada (amarillo) y grave (rojo).

Se evaluó la normalidad de los datos con el estadístico de prueba ShapiroWilk ( $\mathrm{gl}=28, \mathrm{p}=0.082$ para el puntaje inicial, $\mathrm{y} \mathrm{p}=0.100$ en el puntaje final), encontrando que la distribución es normal, por lo que se aplicó el estadístico de prueba T-student, determinándose que la diferencia es estadísticamente significativa $(\mathrm{t}=-10.234, \mathrm{gl}=27, \mathrm{p}=0.000)$. Respecto a la prevalencia del primer diagnóstico, se obtuvo que el $10.7 \%$ de los pacientes presentaron LFM grave, $64.3 \%$ LFM moderada, $21.4 \%$ con LFM leve, y un $3.6 \%$ con LFM mínima. Al término del programa de terapia manual por el método OMT Kaltenborn-Evjenth la LFM predominante fue la leve en un $75 \%$ de los 
pacientes, $10.7 \%$ moderada, y $14.3 \%$ mínima (Figura 2). Denotando con ello la disminución en la LFM en todos los pacientes.

\begin{tabular}{|c|c|c|c|c|c|c|c|c|c|}
\hline & \multicolumn{8}{|c|}{ SPPB1 Puntuación Total Final } \\
\hline & & \multicolumn{2}{|c|}{ Moderada } & \multicolumn{2}{|c|}{ Leve } & \multicolumn{2}{|c|}{ Mínima } & \multicolumn{2}{|c|}{ Total } \\
\hline & & Fr & $\%$ & $\mathrm{Fr}$ & $\%$ & Fr & $\%$ & Fr & $\%$ \\
\hline \multirow{4}{*}{$\begin{array}{c}\text { SPPBI } \\
\text { Puntuación } \\
\text { Total Inicial }\end{array}$} & Grave & 0 & 0.0 & 2 & 7.1 & 1 & 3.6 & 3 & 10.7 \\
\hline & Moderada & 3 & 10.7 & 13 & 46.4 & 2 & 7.1 & 18 & 64.3 \\
\hline & Leve & 0 & 0.0 & 6 & 21.4 & 0 & 0.0 & 6 & 21.4 \\
\hline & Minima & 0 & 0.0 & 0 & 0.0 & 1 & 3.6 & 1 & 3.6 \\
\hline & Total & 3 & 10.7 & 21 & 75 & 4 & 14.3 & 28 & 100 \\
\hline
\end{tabular}

Figura 2. Tabla de contingencia SPPB Puntuación Total Inicial-Puntuación Total Final Presentación de la prevalencia de la clasificación del primer diagnóstico al inicio del tratamiento y al término del programa de terapia manual por el método OMT Kaltenborn-

Evjenth.

\section{Discusión}

Estudios previos exponen las condiciones de los principales problemas en este tipo de pacientes puesto que el proceso de envejecimiento va generando cambios en las estructuras anatómicas condicionando la biomecánica y modificando la marcha en el anciano (Landinez-Parra et al., 2012). Respecto a su abordaje fisioterapéutico existen estudios enfocados a la neurorehabilitación, disminución del dolor, alteraciones podológicas, sedentarismo y al impacto sobre las actividades de la vida diaria (Franco 2017).

Es de suma importancia que este tipo de individuos sean intervenidos fisioterapéuticamente de manera específica a mejorar y/o conservar su marcha funcional debido a que existen alteraciones biomecánicas y funcionales que provocan desequilibrio muscular, atrofias y deformidades estructurales del pie (Grajales-Toro 2016).

El propósito del presente estudio fue demostrar la efectividad del tratamiento fisioterapéutico en la limitación funcional de la marcha en pacientes geriátricos diabéticos dejando constancia de la importancia que tiene la terapia manual ortopédica Kaltenborn-Evjenth para la obtención de resultados óptimos. No se encuentra en la bibliografía ningún estudio semejante al presentado con el que se pueda comparar los resultados obtenidos. No obstante, existen referencias bibliográficas, como las de Pawel Lizis et al (2010) y Wnuk et al (2017), que muestran el alcance de la OMT Kaltenborn-Evjenth en pacientes con escoliosis y discopatía lumbar, resultando favorecedora en el aumento de movilidad y mejora de calidad de vida y dolor.

Durante y al final del tratamiento, sin ser una variable propia del mismo, se pudo observar que el dolor disminuyó, lo cual contribuyó a la mejora de la marcha funcional. La importancia de este estudio es el alcance y fácil accesibilidad que ofrece la terapia OMT Kaltenborn-Evjenth, es una 
técnica que no requiere altas inversiones económicas al aplicarse, el diagnóstico es rápido y los resultados bastante favorecedores. La principal limitación fue el corto período de seguimiento y tamaño de muestra pequeña, por lo que resulta interesante plantear en un futuro estudios similares con mayores tamaños de muestra, incluso comparar diferentes conceptos de terapia manual, lo que permitiría llegar a conclusiones más acertadas respecto a este tipo de trabajo.

\section{Conclusión}

La terapia manual Kaltenborn-Evjenth resultó ser efectiva en el aumento de rangos de movimientos favoreciendo a la cápsula articular y ligamentos. Beneficiando el juego articular, la calidad de vida y disminución del dolor. Esto a su vez, condiciona una mejora en las variables de la funcionalidad de la marcha en la población geriátrica estudiada.

Todos estos resultados pueden ser valiosos para fisioterapeutas y médicos al elegir los tipos de tratamiento más adecuados según las preferencias y a conveniencia de los pacientes con esta condición.

\section{References:}

1. Franco, I. S. (2017). Abordaje fisioterapéutico en personas con diabetes mellitus tipo II: de la revisión científica a la práctica rehabilitadora desde un enfoque neurorrehabilitador. Revista Colombiana de Medicina Física y Rehabilitación, 27(1), 83-97.

2. García-García, José Antonio; Reding-Bernal, Arturo; LópezAlvarenga, Juan Carlos (2013). Cálculo del tamaño de la muestra en investigación en educación médica. Investigación en educación médica 2(8), 217-224.

3. Grajales-Toro, S. (2016). Alteraciones de la marcha en pacientes con diabetes mellitus tipo 2: una revisión de tema (Doctoral dissertation).

4. Hammad, S. M., Arsh, A., Iqbal, M., Khan, W., Bilal, M., \& Shah, A. (2019). Comparing the effectiveness of kaltenborn mobilization with thermotherapy versus kaltenborn mobilization alone in patients with frozen shoulder [adhesive capsulitis]: A randomized control trial. JPMA. The Journal of the Pakistan Medical Association, 69(10), 1421.

5. Herrero-Larrea, A. (2017). Estudio de los parámetros espaciales de la marcha en la población anciana española y su asociación con resultados adversos de salud. (Doctoral dissertation).

6. Kaltenborn, F. M., Evjenth, O., Kaltenborn, T. B., \& Vollowitz, E. (2001). Fisioterapia manual: extremidades. McGraw-Hill Interamericana. 
7. Kang, G. E., Nguyen, H., Zahiri, M., Zhou, H., Wang, C., Goel, R., \& Najafi, B. (2019). Characteristics of the gait initiation phase in older adults with diabetic peripheral neuropathy. Innovation in Aging, 3(474-485).

8. Landinez-Parra, N. S., Contreras Valencia, K., \& Castro Villamil, Á. (2012). Proceso de envejecimiento, ejercicio y fisioterapia. Revista cubana de salud pública, 38, 562-580.

9. Lauretani, F., Ticinesi, A., Gionti, L., Prati, B., Nouvenne, A., Tana, C., \& Maggio, M. (2019). Short-Physical Performance Battery (SPPB) score is associated with falls in older outpatients. Aging clinical and experimental research, 31(10), 1435-1442.

10. Lizis, P., Wiater, S., \& Kobza, W. (2017). Manual Therapy vs. Kinesiotherapy for People with Lumbar Discopathy: A Pilot Randomized Trial. Rehabilitation, 2(1), 6-1.

11. Prishila Danae, R., Enrique, V., Martha Leticia, M., Jorge, V., Nicolás, C., \& Lilia Susana, G. et al. (2016). Costo-Efectividad De Exploración De Miembros Pélvicos Para Prevenir Pie Diabético. European $\begin{array}{lll}\text { Scientific Journal, } & \text { ESJ, 12(24), }\end{array}$ https://doi.org/10.19044/esj.2016.v12n24p137

12. Rodríguez-Mañas, L., Bayer, A. J., Kelly, M., Zeyfang, A., Izquierdo, M., Laosa, O., \& Sinclair, A. J. (2014). An evaluation of the effectiveness of a multi-modal intervention in frail and pre-frail older people with type 2 diabetes-the MID-Frail study: study protocol for a randomised controlled trial. Trials, 15(1), 34.

13. Secretaría de Salud. (2013). Diagnóstico y Tratamiento de Diabetes Mellitus en el Adulto Mayor Vulnerable. México.

14. Sgaravatti, A., Santos, D., Bermúdez, G., \& Barboza, A. (2018). Velocidad de marcha del adulto mayor funcionalmente saludable. Anales De La Facultad De Medicina, 5(2), 93-101. doi: 10.25184/anfamed2018v5n2a8

15. Wnuk, B., Durmala, J., Dzierzega, J., Dybula, K., \& Wadolowski, K. (2010). Short-term effects of simultaneous using of two physiotherapeutic methods in the treatment of adolescent idiopathic scoliosis-continuation of study? Scoliosis, 5(1), 27-44. 